\title{
Wafer-scale fabricated thermo-pneumatically tunable microlenses
}

\author{
Wei Zhang, Hans Zappe and Andreas Seifert
}

We have developed a self-contained, liquid tunable microlens based on polyacrylate membranes integrated with compact on-chip thermo-pneumatic actuation fabricated using full-wafer processing. Silicone oil is used as the optical liquid, which is pushed or pulled into the lens cavity via an extended microfluidic channel structure without any pumps, valves or other mechanical means. The heat load generated by the thermal actuator is physically isolated from the lens chamber. The back focal length may be tuned from infinity to $4 \mathrm{~mm}$ with a maximum power consumption of $300 \mathrm{~mW}$. The principal application is fine tuning of the back focal length, for which tuning time constants as small as $100 \mathrm{~ms}$ are suitable.

Light: Science \& Applications (2014) 3, e145; doi:10.1038/lsa.2014.26; published online 28 February 2014

Keywords: optofluidics; thermo-pneumatic actuation; tunable microlens; wafer-scale fabrication

\section{INTRODUCTION}

Miniaturization of tunable lenses for integration into optically reconfigurable optical microsystems is promising for many applications, including optical communications, consumer electronics and medical


technology ${ }^{2}$ are available commercially; however, high voltages are typically required in such systems. Polydimethylsiloxane membrane lenses, operated by pumping an optical liquid into or out of a lens cavity, are of interest because of their low fabrication cost and easy implementation of a variety of actuation mechanisms, ${ }^{3}$ such as pneumatic, piezoelectric, ${ }^{4}$ electromagnetic ${ }^{5,6}$ and thermal actuators. ${ }^{7}$ However, limitations in the selection of liquid and membrane materials restrict the development of new actuation mechanisms, particularly from the standpoint of wafer-level microelectromechanical systems manufacturing processes.

We present here a completely self-contained, thermo-pneumatically tunable microlens with on-chip actuation, fabricated using microelectromechanical systems techniques in a full-wafer fabrication process. The tunability is achieved by heating air in a pressure-generating chamber, which is connected via a meander-shaped channel to a liquid-filled optical chamber bounded by a polyacrylate membrane without using any actuation membranes, valves or mechanically movable parts. This method benefits from low driving voltages, but yields high pressures, inducing a large deflection of the surface of the tunable lens resulting in a wide focal length tuning range.

In comparison to previous work on integrated thermo-pneumatically tunable microlenses, ${ }^{8}$ the concept presented here is considerably more compact and robust, with significantly improved thermal isolation between the actuator and the optical path. In addition, the present concept allows wafer-level fabrication, implying a lower potential cost due to improved yield.

\section{LENS DESIGN}

Figure 1a presents an exploded view of the fully integrated thermopneumatic microlens, which consists of five major parts; the corresponding thicknesses are given in parentheses. From top to bottom, these are: a silicon lens frame $(500 \mu \mathrm{m})$, a silicon lens chip $(300 \mu \mathrm{m})$, a flexible polyacrylate membrane $(50 \mu \mathrm{m}),{ }^{9}$ a dry film resist (DFR; Ordyl) layer forming microfluidic structures and a glass substrate $(500 \mu \mathrm{m})$ with heating and temperature sensing structures. Three rectangular openings are present in the silicon lens frame: one for filling the lens cavity with the optical liquid, one as a vent for removing air and the third for centering the silicon lens chip, which has a circular aperture $2 \mathrm{~mm}$ in diameter. A thin polyacrylate membrane, which defines the refractive interface of the lens, is sandwiched between the silicon lens chip and the fluid chamber. Using this approach, stable bonding is attained, and delamination of the membrane at the margins is prevented, even for the case of strong membrane deformation.

The working principle of the microlens is based on the thermal expansion of air in the air chamber. As shown in Figure 1b, the air chamber is directly connected to the lens cavity via a microchannel that is filled with silicone oil as the optical liquid. When a voltage is applied to the heater, the rising temperature causes a volume increase, and the expanding air pumps the silicone oil through the meandershaped microchannel into the lens cavity. Because the liquid can be assumed to be incompressible, the volume increase of the expanded air will cause deformation of the polyacrylate lens membrane, thereby tuning the back focal length (BFL) of the lens.

The dimensions of the air chamber and the channel structure are selected such that the expected volume displacement corresponds to the required BFL tuning range. The shortest BFL determines the required dimensions. In the present realization, the shortest 


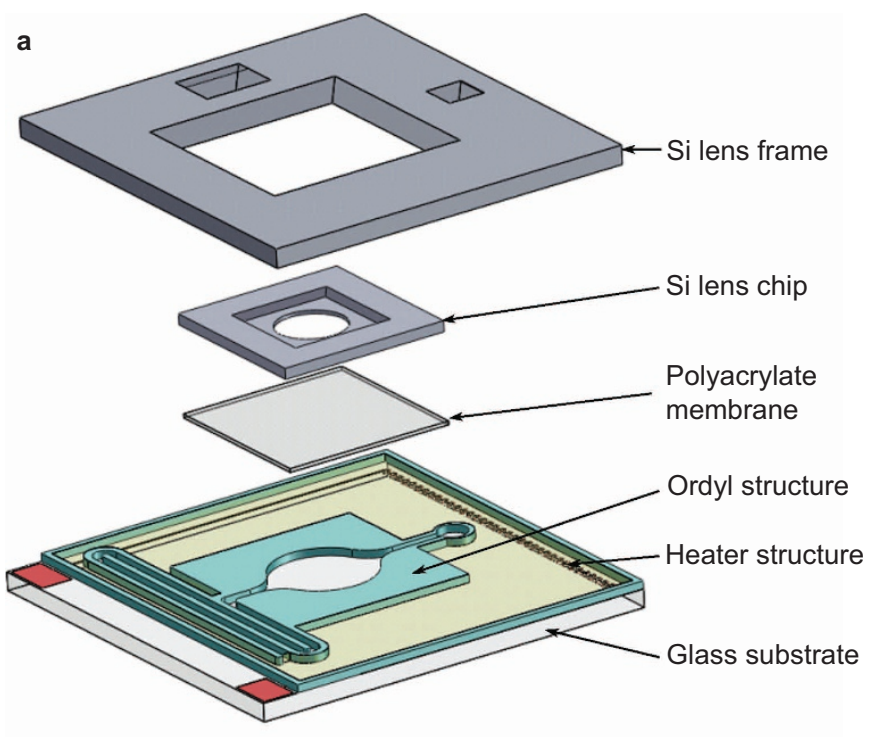

b

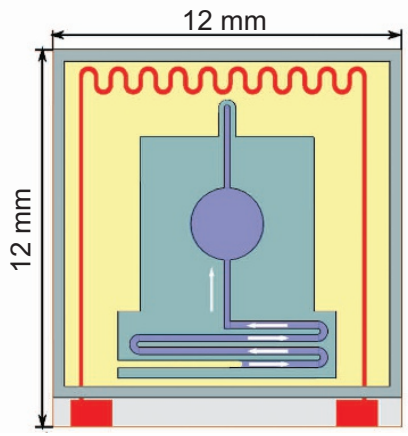

Figure 1 (a) Exploded view of the fully integrated thermo-pneumatic microlens. The red square areas at the left margin of the glass substrate are the electrical contact pads for the heater, which is on the opposite side of the glass chip (the wavy red line). (b) Top view of the microfluidic design. The liquid chamber and channel structure are filled with silicone oil (blue), and the outer larger chamber is filled with air (yellow). By heating the air, its volume increases and displaces the optical liquid in the channel. The displaced liquid is pushed into the lens cavity, thereby deflecting the polymer membrane, resulting in a change in the refractive power. appropriate BFL is approximately $5 \mathrm{~mm}$. For a $2-\mathrm{mm}$ diameter lens using silicone oil as the liquid with a refractive index of 1.4 , the corresponding volume of the spherical lens cap is approximately $0.5 \mathrm{~mm}^{3}$.

The expanded air volume is not expected to directly transfer the same volume displacement to the optical cavity because air is a compressible medium. Thus, all repulsive forces-for example, capillary forces, viscosity or the repulsive forces of the membrane-will compress the air volume, leading to a small net volume change. Therefore, a buffer must be included in the fluidic layout. The designed lens has an air chamber volume of approximately $14 \mathrm{~mm}^{3}$; the theoretical thermal expansion of air at constant pressure is $\Delta V=1.4 \mathrm{~mm}^{3}$ for a temperature shift of $\Delta T=30 \mathrm{~K}$, and the cross-section of the fluidic channel is $0.25 \mathrm{~mm} \times 0.22 \mathrm{~mm}$. Thus, to provide a volume expansion of $1.4 \mathrm{~mm}^{3}$, a channel length of $25 \mathrm{~mm}$ is required. Owing to the small size of the microchannel and the resulting low Reynolds number, this device is resistant to shock and vibration during lens operation, and no liquid back flow occurs because capillary forces, surface tension and viscous forces dominate over gravitational forces in the laminar flow.

\section{THERMAL SIMULATION}

Because temperature gradients introduce refractive index variations in optical components, the temperature distribution in the optical liquid must be highly homogeneous to ensure good imaging quality. A novel feature of the structure presented here is that the optical lens liquid is not heated directly, but is thermally insulated from the heater by the microchannel structures. We evaluate the resulting thermal homogeneity of the lens using multiphysics simulation software (COMSOL 3.5a, USA). The Conductive Media DC module from AC/DC model, and the heat transfer module which was defined to allow thermal convection outside the chip were employed for the boundary conditions. For the heater structure in the model, a 150-nm-thick platinum layer was embedded as a two-dimensional structure on the surface of the glass substrate.

Figure 2 shows the modeled three-dimensional structure, consisting of a circular meander-shaped heater, the air volume trapped in the air chamber, the microchannel, the optical liquid filled in the lens cavity and the microchannel, the silicon bulk structures and the lens membrane. The temperature distribution of the lens structure is calculated for a bias of $8.5 \mathrm{~V}$-corresponding to approximately $180 \mathrm{~mW}$ applied to the heater. From this result, we conclude that the temperature homogeneity across the lens structure is determined mainly by the

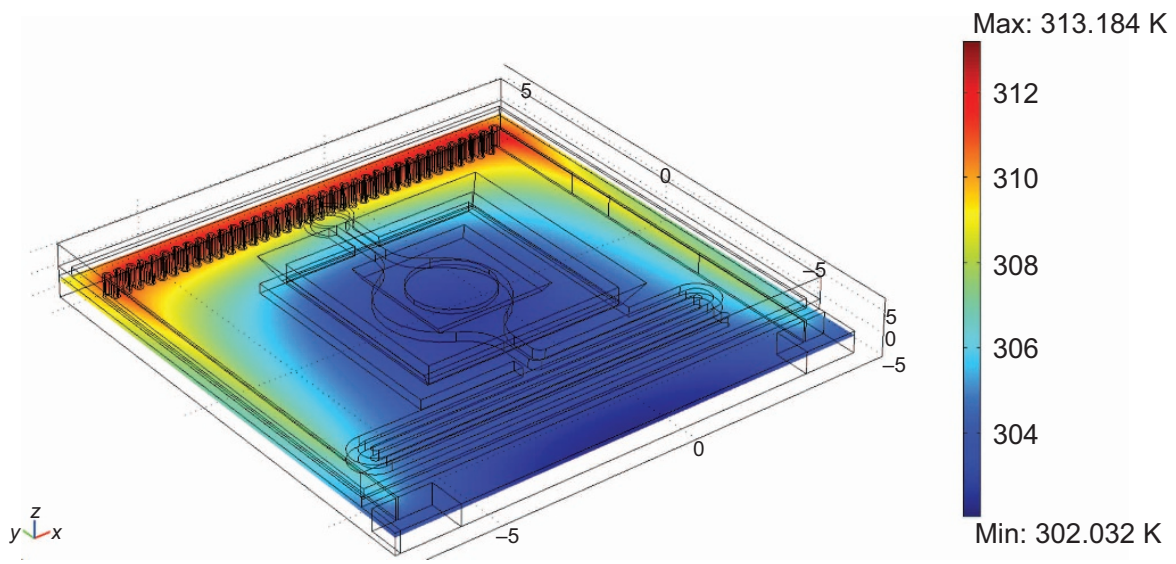


The surrounding temperature is $293 \mathrm{~K}$. FEM, finite element method. 

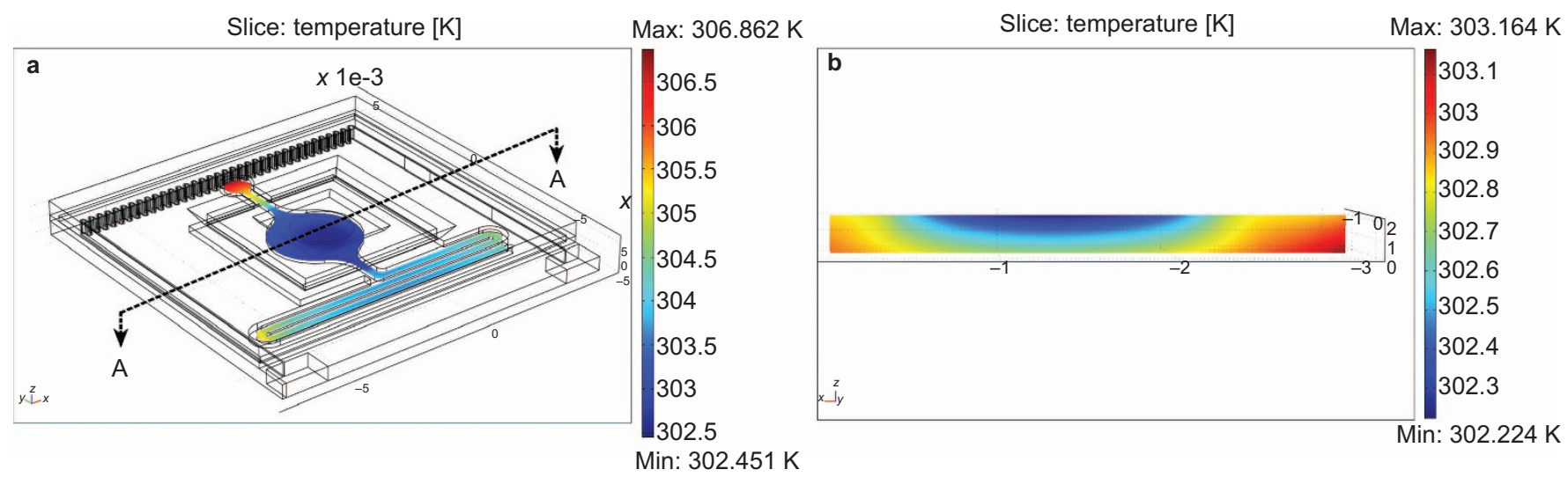

Figure 3 FEM-simulation results: temperature distribution in the optical liquid in (a) the horizontal direction at the bottom of the liquid and (b) the vertical direction.

geometry of the heater. Accordingly, the heater structures were arranged symmetrically around the optical liquid in the final design.

The temperature homogeneity of the optical liquid is investigated in further detail in Figure 3. In Figure 3a, the temperature variation across the lens structure is less than $4 \mathrm{~K}$ in the horizontal direction and is concentrated primarily at the liquid inlet near the heater structure. In the optical path, the temperature varies by less than $0.2 \mathrm{~K}$. In the vertical direction, as shown in Figure $3 \mathrm{~b}$, the temperature variation within the ultrathin silicone oil film is less than $0.5 \mathrm{~K}$. These values are attained because the trapped air around the Ordyl structures is thermally insulating due to its low coefficient of thermal conductivity $\left(0.025 \mathrm{~m}^{-1} \mathrm{~K}^{-1}\right)$.

\section{FABRICATION TECHNOLOGY}

The microfluidic structures defining the air chamber, optical cavity and connecting microchannel are fabricated using the DFR Ordyl SY550 with a thickness of $55 \mu \mathrm{m}$ (Elga Europe, Italy). ${ }^{10}$ The process chain is illustrated in Figure 4. First, a 20-nm-thick binding layer of titanium and a 150-nm-thick layer of platinum are deposited on the glass wafer using electron beam evaporation and are patterned using a lift-off process to define the heating and temperature-sensing elements, as well as the alignment structures. The size of the heater structure is limited to widths of approximately $50 \mu \mathrm{m}$. Next, four layers of Ordyl SY550 are laminated onto the glass wafer using a desktop laminator at a temperature of $90-100{ }^{\circ} \mathrm{C}$. After lamination, the DFR is exposed with a mask aligner (MA6; Süss Microtec, Germany) with a dose of $315 \mathrm{~mJ} \mathrm{~cm}{ }^{-2}$ and then baked at $85{ }^{\circ} \mathrm{C}$ for $2 \mathrm{~min}$ on a



Figure 4 Process flow for the lens frame. (a) Pt lift-off; (b) hot roll lamination; (c) lithography; (d) DFR developing; (e) Si wafer with $\mathrm{KOH}$ wet etched cavities; (f) full wafer bonding. DFR, dry film resist. hotplate to initiate crosslinking. The wafer is subsequently developed in BMR developer for $5 \mathrm{~min}$.

Figure $5 \mathrm{a}$ is a photograph of the fabricated glass wafer after lift-off and DFR development. In Figure 5b, we see that, for each chip, the DFR forms a rectangular frame with $12 \mathrm{~mm} \times 11 \mathrm{~mm}$, microchannels with dimensions of $25 \mathrm{~mm} \times 0.22 \mathrm{~mm} \times 0.25 \mathrm{~mm}$ and a circular chamber for the optical liquid with a diameter of $3 \mathrm{~mm}$. The ridges of the outer Ordyl confinement have a width of $400 \mu \mathrm{m}$. To seal the air chamber and channel structures, the Si wafer with the lens frames is
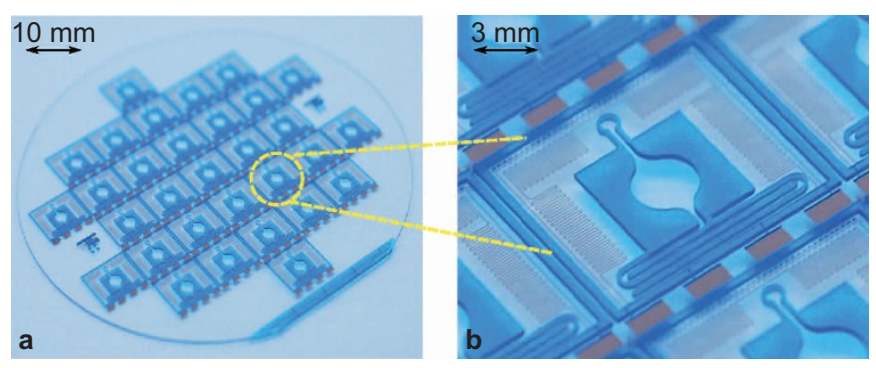

Figure 5 (a) Photograph of a glass wafer prepared for wafer bonding. (b) Detailed view of the DFR structure showing a single chip cell. DFR, dry film resist.

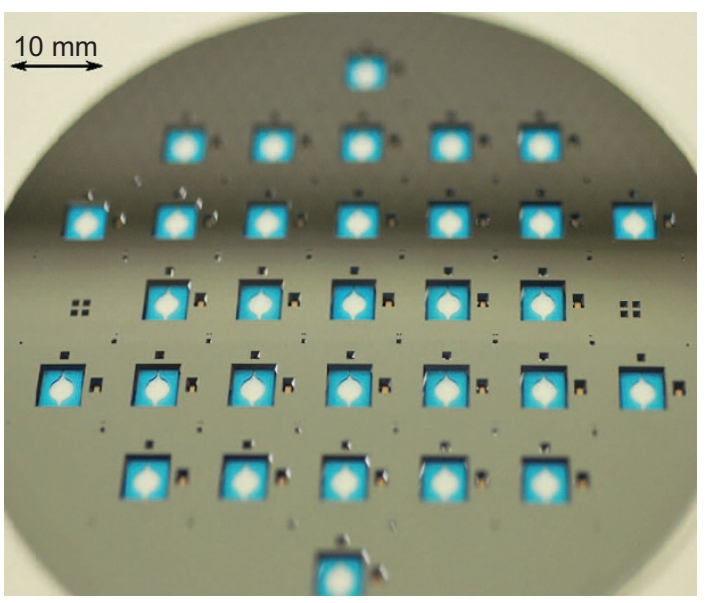

Figure 6 Photograph of the etched Si wafer bonded with the glass wafer with an intermediate dry film resist layer. The 4-inch wafer comprises 31 devices. A detailed view of the device is presented in Figure 7. 

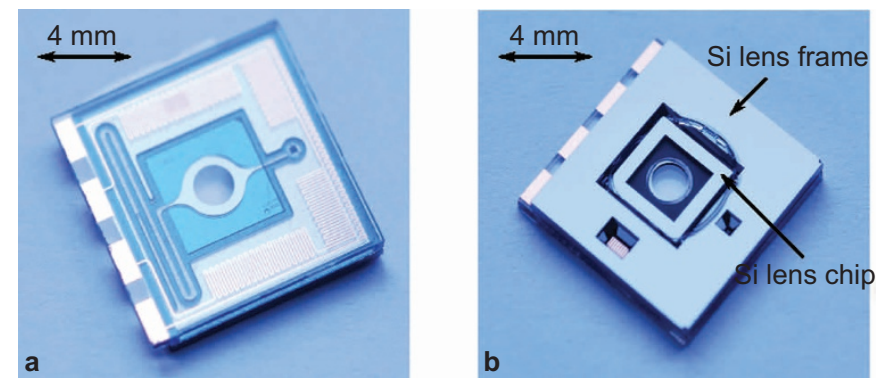

Figure 7 (a) Photograph of the back of the bonded Si lens chip with microfluidic structures. (b) Photograph of the fully integrated lens device; the membrane is sandwiched between the rectangular Si lens chip and the Ordyl structures beneath.

bonded onto the Ordyl configuration and thus also bonds with the narrow side ridges.

The silicon components are finished by standard silicon bulk micromachining. Three cavities of different sizes are etched into the Si cover frame: the lens cavity of $5.85 \mathrm{~mm} \times 5.85 \mathrm{~mm}$, a liquid inlet opening of $1.1 \mathrm{~mm} \times 1.1 \mathrm{~mm}$ and an air vent of $1.5 \mathrm{~mm} \times 2 \mathrm{~mm}$. These openings are achieved by anisotropic $\mathrm{KOH}$ wet etching on the back of the Si wafer. The two wafers are aligned and bonded together using the DFR structure and a wafer bonder (SB6; Süss Microtec, Germany) under a pressure of $1400 \mathrm{mbar}$ at $90{ }^{\circ} \mathrm{C}$ for $30 \mathrm{~min}$.

Figure 6 shows the bonded stack with a total height of $1.22 \mathrm{~mm}$, consisting of a glass wafer, the internal fluidic structures with a thickness of $220 \mu \mathrm{m}$ and the Si wafer defining the lens frames. In the large openings of the lens frames, DFR areas onto which the silicon lens chips are later attached with the polyacrylate membrane in-between are visible. As a final step, the wafer is diced to open the electrical contact pads and separate the single lens elements.

\section{FINAL ASSEMBLY AND LENS FILLING}

In the final assembly step, the adhesive polyacrylate membrane is attached to the bottom side of the silicon lens chip. This hybrid element is positioned in the central large opening of the silicon frame and bonded using the adhesive properties of the polyacrylate with slight pressure on the accessible Ordyl structures in the opening. The opening is $30 \mu \mathrm{m}$ larger than the $\mathrm{Si}$ lens chip. The original polyacrylate tape (3M, VBH4905) has a thickness of $500 \mu \mathrm{m}$ and poor optical performance. ${ }^{11,12}$ To improve optical properties such as transparency and microroughness, the tape is thinned to $50-100 \mu \mathrm{m}$. This is done by inflating the tape, yielding a low and homogeneously distributed stress. $^{9,13}$

The assembled device is shown in Figure 7. To prevent liquid leakage, an ultraviolet-curable epoxy is applied to permanently seal the gap between the lens chip and the lens frame. The fluidic structure with the microchannel connecting the air chamber with the optical cavity can be clearly seen in the left panel of Figure 7 .

In many designs, additional external pressure or vacuum must be applied to allow filling of the liquid device. ${ }^{14}$ However, in the present work, low surface tension silicone oil was employed, resulting in a selffilling procedure employing only capillary forces, which ensures a bubble-free filling process of the microfluidic structures. ${ }^{15}$ The device can thus be filled without using pressure tubes or vacuum.

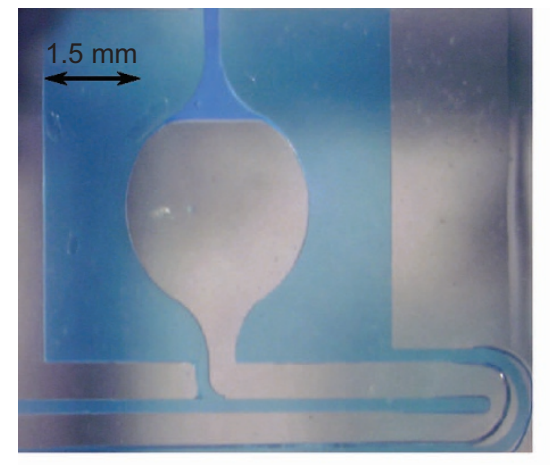

$4 \mathrm{~s}$



$8 \mathrm{~s}$

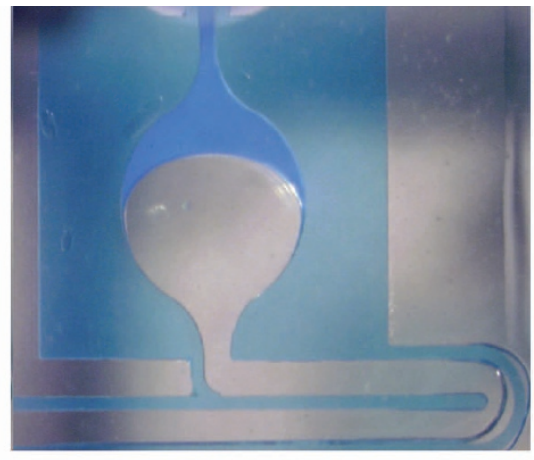

$6 \mathrm{~s}$

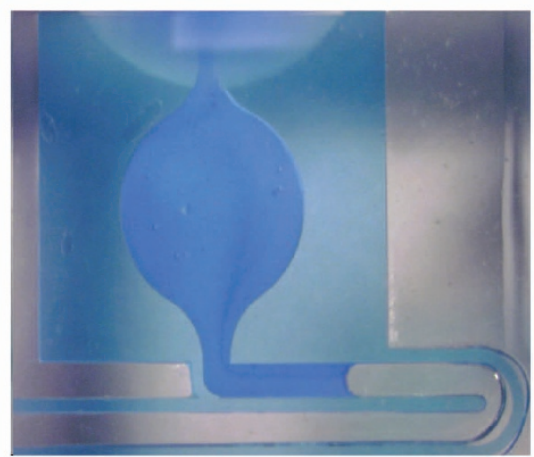

$12 \mathrm{~s}$

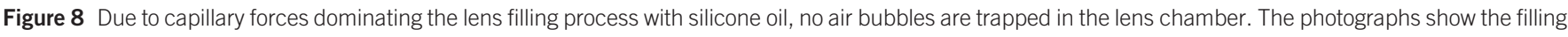

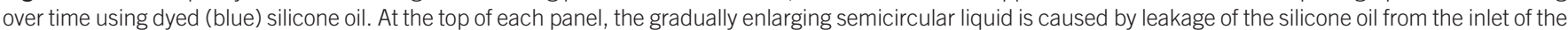
PMMA plate. The inlet is not visible. PMMA, poly (methyl methacrylate). 
To prevent silicone oil from entering the air chamber, a small droplet of the oil with a volume of $2.2 \mu \mathrm{L}$ was injected using a micropipette through the liquid inlet. The droplet completely fills the optical chamber and creeps into the microchannel due to capillary forces. The droplet volume is dimensioned such that three-fourths of the channel length is filled. Because the forces are balanced, the filling stops automatically when the droplet has been completely injected. After filling, the inlet is sealed.

The filling process is demonstrated using a test device consisting of a flat poly(methyl methacrylate) substrate with a fluidic inlet and a DFR fluidic network patterned on a glass substrate; polyacrylate tape is used to attach both parts together. To make the dynamic filling process observable, the chamber is filled with blue-dyed silicone oil with a viscosity of $50 \mathrm{cSt}$. The temporal development of the filling is depicted in Figure 8. Again, no additional external pressure was applied from the liquid inlet. Driven by capillary forces, the silicone oil automatically flows from the inlet into the lens chamber and the microchannel without trapping any air bubbles.

\section{RESULTS AND DISCUSSION}

\section{BFL}

The BFL was measured by determining the position of the circle of least confusion, using a microscopic setup with collimated white light in front of the tunable lens. ${ }^{16}$ By applying suitable voltages to the heating unit of the lens, the BFL can be adjusted, as illustrated in Figure 9. The relative temperature is measured using a sensor integrated on the chip, which was calibrated by a high-accuracy Pt100 temperature sensor. Figure 9 shows the results of the BFL measurement and the corresponding temperature, which was measured at thermal equilibrium using commercial Pt100 temperature sensors, both as a function of the power consumption. The plot indicates that BFL can be tuned with good repeatability and that only minimal hysteresis occurs between the heating and cooling ramps. Due to a slight overlap of the error bars, the deviation between the curves might

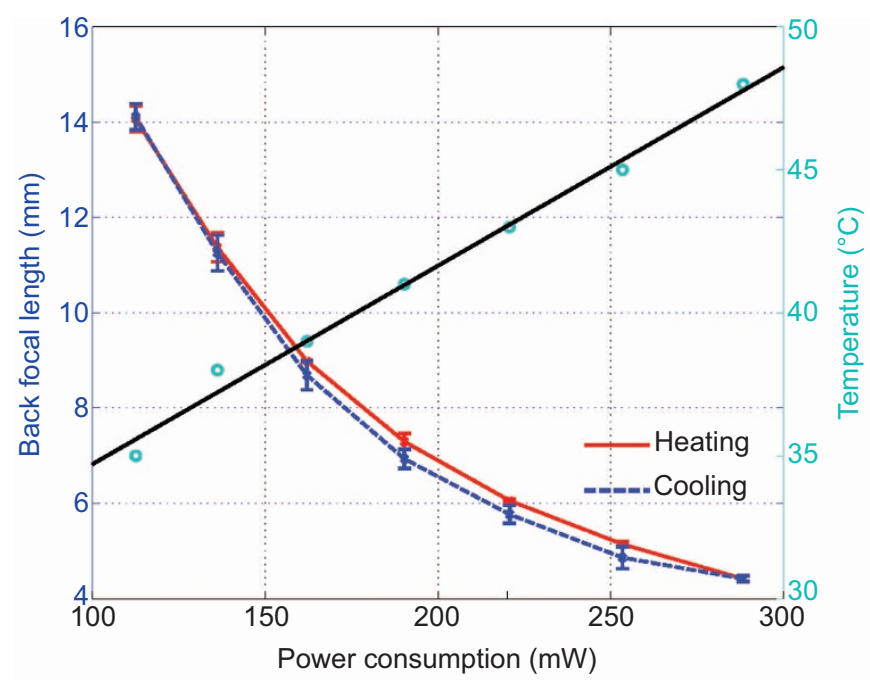

Figure 9 BFL and temperature of the lens with respect to power consumption of the heater. The red and blue least-squares fits show the BFL behavior for heating and cooling, respectively. The maximum hysteresis between both curves is $\leqslant 0.5 \mathrm{~mm}$ in back focal length and might be caused by different response times of heating and cooling. The black line is a linear fit through the corresponding temperature values, indicating the linear behavior of the temperature with power consumption. BFL, back focal length. be negligible. The corresponding voltage range for this lens device was below $7.5 \mathrm{~V}$.

\section{Modulation transfer function (MTF) and imaging}

The dynamic tuning of the lens and the corresponding imaging quality are shown in Figure 10, which presents four snapshots of an imaged 'F'-structure at an object distance of $40 \mathrm{~mm}$. The images are magnified by an additional microscope objective. At zero voltage, the membrane of the lens is flat and the recorded image from the CCD is the same size as the object, under the magnification of the objective lens. After the lens has been heated for $30 \mathrm{~s}$ with an electric power of $150 \mathrm{~mW}$, a stable magnified image is observed, as can be seen in the upper right panel of Figure 10. To reduce the refractive power, the system must be cooled passively, simply by switching off the power. The two lower panels in Figure 10 are snapshots taken after 13 and 43 s of cooling, respectively. The original image size is reached again after $43 \mathrm{~s}$.

The BFL tuning demonstrates that the expected time constants are on the order of tens of seconds. However, the lens is not intended for applications that require tuning over a large BFL range, but rather for focal length correction in an optical system with other, fixed-focus elements. A suitable tuning range is between 5 and $20 \mathrm{~mm}$, for which time constants of several hundred milliseconds apply.

For a quantitative analysis of the image quality, the MTF of the microlens was determined from the point spread function using the same set-up. ${ }^{16}$ Figure 11 shows four different MTF curves measured at different actuation states and the corresponding diffraction-limited MTFs. At a reference contrast of 0.2 , the cutoff frequency varies between 46 and 75 lines $\mathrm{mm}^{-1}$ over the BFL tuning range from 5.3 to $11.5 \mathrm{~mm}$. The best MTF performance is achieved at the largest BFL of $11.5 \mathrm{~mm}$ (NA 0.09), which corresponds to $38^{\circ} \mathrm{C}$ or an electric power of $135 \mathrm{~mW}$. With larger deformation of the lens membrane, the MTF of the lens decreases. Basically, the MTF should increase with increasing numerical aperture; however, aspherical deformations, which


Figure 10 Four snapshots demonstrating the tunability of the microlens, taken during heating and cooling. An object with an 'F'-structure is imaged at different actuation states. After one thermal cycle, the original image occurs again (first and last pictures). The dashed circle in the lower part of each photograph indicates the position of the liquid/air interface. 




Figure 11 MTF curves of the microlens measured at different back focal lengths. The corresponding temperature and power consumption can be determined from Figure 9. The best MTF performance is obtained at a back focal length of $11.5 \mathrm{~mm}$. With increasing deformation of the membrane and decreasing back focal length, MTF performance diminishes continuously. MTF, modulation transfer function.

cause aberrations, become increasingly prominent at shorter BFLs and reduce the contrast. The MTF results agree well with the images in Figure 10.

\section{CONCLUSIONS}

We introduce a novel approach for the fabrication of a fully integrated thermo-pneumatic microlens. The experimental results demonstrate that the BFL of the microlens can be tuned with high accuracy and excellent repeatability due to a microfluidic network. The device features several new and interesting properties: integration of the tunable lens with a microfluidic channel, which connects the optical cavity with a heated air reservoir without any movable mechanical parts; high-yield wafer level processing; a small chip size $(12 \mathrm{~mm} \times 12 \mathrm{~mm} \times 1.22 \mathrm{~mm})$; a variable lens size without changing the fabrication technology; and the absence of liquid evaporation and convenient filling into the ultrathin lens chamber without trapping air bubbles, using a polyacrylate membrane and silicone oil. Another distinct advantage is the minimized thermal cross-talk between the heated air chamber and the optical cavity due to the symmetric design and use of air as the pumping medium.

1 Kuiper S, Hendriks BH. Variable-focus liquid lens for miniature cameras. Appl Phys Lett 2004; 85: 1128-1130.

2 Ren H, Wu ST. Adaptive liquid crystal lens with large focal length tenability. Opt Express 2006; 14: 11292-11298.

3 Werber A, Zappe H. Tunable microfluidic microlenses. Appl Opt 2005; 16: 32383245 .
4 Schneider F, Draheim J, Kamberger M, Waibel P, Wallrabe U. Optical characterization of adaptive fluidic silicone-membrane lenses. Opt Express 2009; 17: 11813-11821.

5 Lee SW, Lee SS. Focal tunable liquid lens integrated with an electromagnetic actuator. Appl Phys Lett 2007; 90: 121129.

6 Mader D, Marhöfer M, Waibel P, Zappe H, Seifert A. Tunable micro-fluidic multicomponent micro-lens system with integrated actuator. In: Proceedings of IEEE 23rd International Conference on Micro Electro Mechanical Systems (MEMS); 24 28 January 2010; Wanchai, Hong Kong. IEEE Robotics and Automation Society: Piscataway, NJ, USA, 2010, pp 799-802.

7 Wang W, Ji F. Design, fabrication and testing of a micromachined integrated tunable microlens. J Micromech Microeng 2006; 16: 1221-1226.

8 Zhang W, Aljasem K, Zappe H, Seifert A. Completely integrated, thermopneumatically tunable microlens. Opt Express 2011; 19: 2347-2362.

9 Zhang W, Zappe H, Seifert A. Polyacrylate membrane for tunable liquid-filled microlenses. Opt Eng 2013; 52: 046601.

10 Müller P, Spengler N, Zappe H, Mönch W. An optofluidic concept for a tunable microiris. J Microelectromech Syst 2010; 19: 1477-1484.

11 Pelrine R, Kornbluh R, Pei QB, Joseph J. High-speed electrically actuated elastomers with strain greater than 100\%. Science 2000; 287: 836-839.

12 Michel S, Zhang XQ, Wissler M, Loewe Q, Kovacs G. A comparison between silicone and acrylic elastomers as dielectric materials in electroactive polymer actuators. Polym Int 2010; 3: 391-399.

13 Cho SH, Tsai FS, Qiao W, Kim NH, Lo YH. Fabrication of aspherical polymer lenses using a tunable liquid-filled mold. Opt Lett 2009; 34: 605-607.

14 Monahan J, Gewirth AA, Nuzzo RG. A method for filling complex polymeric microfluidic devices and arrays. Anal Chem 2001; 13: 3193-3197.

15 Kang JH, Kim YC, Park JK. Analysis of pressure-driven air bubble elimination in a microfluidic device. Lab Chip 2008; 8: 176-178.

16 Zhang W, Aljasem K, Zappe H, Seifert A. Highly flexible MTF measurement system for tunable micro lenses. Opt Express 2010; 18: 12458-12469.

\footnotetext{
(c) (i) $\odot$ This work is licensed under a Creative Commons AttributionNonCommercial-NoDerivs Works 3.0 Unported license. To view a copy of this license, visit http://creativecommons.org/licenses/by-nc-nd/3.0
} 